\title{
2014 Scientific Referees
}

(C) Italian Society of Orthopaedics and Traumatology 2015

The quality of the Journal of Orthopaedics and Traumatology depends on the qualified and regular collaboration of renowned scientists who devoted their time to

Mark Adams (Newark, USA)

Roberto Adani (Verona, Italy)

Ezio Adriani (Rome, Italy)

Gabriele Agati (Orbassano, Italy)

Mustafa Alnaib (Tunbridge Wells, UK)

Antonio Andreacchio (Turin, Italy)

Alessandro Aprato (Turin, Italy)

Stefano Artiaco (Turin, Italy)

Andrea Atzei (Verona, Italy)

Francesco Atzori (Turin, Italy)

Angelo Aulisa (Rome, Italy)

Pavan Kumar Avadhanam (Karimnagar, India)

Mostafa Ayoub (Tanta, Egypt)

Giovanni Barbanti Brodano (Bologna, Italy)

Paolo Baudi (Modena, Italy)

Lorenza Belletti (Modena, Italy)

Vittorio Bellotti (Barcelona, Spain)

Teresa Benigno (Turin, Italy)

Federica Bergamin (Turin, Italy)

Giuseppe Bianchi (Bologna, Italy)

Amin Bigham (Shahrekord, Islamic Republic of Iran)

Amedeo Bini (Varese, Italy)

Kristian Bjorgul (Fredrikstad, Norway)

Silvio Luigi Boero (Genoa, Italy)

Filippo Boniforti (Cefalù, Italy)

Andrea Borgo (Padua, Italy)

Mario Borroni (Rozzano, Italy)

Elena Maria Brach del Prever (Turin, Italy)

Roberto Emanuele Buda (Bologna, Italy)

Matteo Cadossi (Bologna, Italy)

Filippo Calderazzi (Parma, Italy)

Domenico Campanacci (Florence, Italy) constructively review the submitted articles. We are indebted to the following experts who reviewed papers which completed the peer-reviewing process within 2014.

Laura Campanacci (Bologna, Italy)

Christian Carulli (Florence, Italy)

Marco Cassini (Legnago, Italy)

Simone Cerciello (Rome, Italy)

Giovanni Ciaramella (Naples, Italy)

Riccardo Ciatti (Rome, Italy)

Norberto Confalonieri (Milan, Italy)

Luigi Conforti (Turin, Italy)

Aurélien Courvoisier (Grenoble, France)

Walter Daghino (Turin, Italy)

Carlo Dall'Oca (Verona, Italy)

Umberto De Bellis (Milan, Italy)

Silvana De Giorgi (Bari, Italy)

Orazio De Lucia (Milan, Italy)

Luigi De Palma (Ancona, Italy)

Ferdinando Del Prete (Florence, Italy)

Giacomo Delle Rose (Rozzano, Italy)

Angelo Dettoni (Turin, Italy)

Shabir A. Dhar (Srinagar, India)

Alberto Di Martino (Rome, Italy)

Francesco Falciglia (Rome, Italy)

Pasquale Farsetti (Rome, Italy)

Francesco Fauci (Cattolica, Italy)

Flavio Fazioli (Naples, Italy)

Andrea Ferretti (Rome, Italy)

Matteo Formica (Genoa, Italy)

Olimpio Galasso (Catanzaro, Italy)

Eduardo García-Rey (Madrid, Spain)

Giorgio Gasparini (Catanzaro, Italy)

Giuseppe Giannicola (Rome, Italy)

Matthew Giordano (Rome, Italy)

Federico Grassi (Novara, Italy) 
Tetsuo Hagino (Kofu, Japan)

Massimo Innocenti (Florence, Italy)

Ernesto Ippolito (Rome, Italy)

Lukas Iselin (Basel, Switzerland)

Nicola Ivaldo (Acqui Terme, Italy)

Ismail Emre Ketenci (Istanbul, Turkey)

Yousuf Khira (Zagazig, Egypt)

Ozkan Kose (Antalya, Turkey)

Antonios Kouzelis (Patras, Greece)

Arun Kumar (Manchester, UK)

Franco Maria Lavini (Verona, Italy)

Andrea Lisai (Milan, Italy)

Giandomenico Logroscino (Rome, Italy)

Giovanni Lovisetti (Menaggio, Italy)

Giulio Maccauro (Rome, Italy)

Bruno Magnan (Verona, Italy)

Leonardo Marchesini Reggiani (Bologna, Italy)

Ignazio Marcoccio (Brescia, Italy)

Alessandra Maresca (Bologna, Italy)

Fabrizio Margheritini (Roma, Italy)

Alessandro Marinelli (Bologna, Italy)

Matteo Marullo (Milan, Italy)

Marcos Matos (Salvador, Brazil)

Barbara Melis (Cagliari, Italy)

Jane Christiane Messina (Milan, Italy)

Luigi Milandri (Ravenna, Italy)

Giovanni Monteleone (Rome, Italy)

Thomasz Nizegorodcew (Rome, Italy)

Masahiko Nozawa (Nerima-ku, Japan)

Alessia Pagnotta (MD) (Rome, Italy)

Hemant Parekh (Pune, India)

Shelain Patel (London, UK)

Marco Pavesi (San Donato Milanese, Italy)

Vito Pavone (Catania, Italy)

Alessio Pedrazzini (Parma, Italy)

Raimondo Piana (Turin, Italy)

Franco Pleitavino (Genoa, Italy)
Enrico Pola (Rome, Italy)

Michele Rampoldi (Roma, Italy)

Enrico Rebuzzi (Oderzo, Italy)

Dario Regis (Verona, Italy)

Olav Reikerås (Oslo, Norway)

Mohammed Riazuddin (Wigan, UK)

Stefano Rigotti (Negrar, Italy)

Giovanni Risitano (Messina, Italy)

E. Carlos Rodriguez-Merchan (Madrid, Spain)

Carlo L. Romanò (Milan, Italy)

Stefano M.P. Rossi (Pavia, Italy)

Claudio Rovesta (Modena, Italy)

Pietro Ruggieri (Bologna, Italy)

Luigi Sabatini (Saluzzo, Italy)

Kabul Saikia (Guwahati, India)

Nicola Santori (Milan, Italy)

Celeste Scotti (Milan, Italy)

Ahmed Siam (Alexandria, Egypt)

Saurabh Singh (Varanasi, India)

Giuseppe Solarino (Bari, Italy)

Naveen Tahasildar (Chandigarh, India)

Luigi Tarallo (Modena, Italy)

Rajiv Thukral (New Delhi, India)

Saket Tibrewal (London, UK)

Davut Tiren (Breda, Netherlands)

Gianpaolo Torasso (Orbassano, Italy)

Federico Trentani (Bologna, Italy)

Cosimo Tudisco (Rome, Italy)

Antonio Vadalà (Rome, Italy)

Gianluca Vadalà (Rome, Italy)

Luca Vaienti (San Donato Milanese, Italy)

Maria Valencia Mora (Madrid, Spain)

Giovanni Vavalle (Bari, Italy)

Paulo Roberto Vilaça Júnior (São Paulo, Brazil)

Parmar Vinay (Manchester, UK)

Giovanni Zatti (Monza, Italy) 